\title{
Ecopuzzles/Evopuzzles: una nueva metodología activa para la enseñanza- aprendizaje. (I) Interacciones ecológicas y (II) Relaciones evolutivas de los Tetrápodos.
}

\section{Ramón González Ruiz}

Dpto Biología Animal, Vegetal y Ecología. Universidad de Jaén.

ramonglz@ujaen.es rglezrui@gmail.com

\begin{abstract}
The ecosystems are self-sufficient habitats where the organisms and the environment exchange energy and matter in a constant cycle. The multiple existing interactions, and the dense flows by which this exchange is canalized (trophic chains) it does of the comprehension of the ecosystems a complex task.

On the other hand, the gradual variation of biological structural complexity and specific diversity in the Animal Kingdom throughout the time, from basal relatively simple forms, up to different lines of vertebrates, - as branches of the evolutionary tree - raises the need to elaborate methodologies that allow to establish the interrelationships among these, as well as with the current extant species.

With this project we have implemented a material and a methodology adapted for the education and spreading of the mentioned relations, by means of a playful, attractive and reasonably intuitive method.
\end{abstract}

Keywords: Mediterranean ecosystems, trophic relationships, evolutionary relationships, predator, parasitoid, pest, Tetrapodes's evolutionary lines, formation, methodology.

\section{Resumen}

Los ecosistemas son hábitats autosuficientes donde los organismos y el ambiente intercambian energía y materia en un ciclo continuo. Las múltiples interacciones existentes, y los intrincados flujos por los que se canaliza este intercambio (cadenas tróficas) hace de la comprensión de los ecosistemas una tarea compleja.

Por otra parte, la variación gradual de la complejidad biológica y de la diversidad en el Reino Animal, desde formas basales relativamente simples, 
Ecopuzzles/Evopuzzles: una nueva metodología activa para la enseñanza-aprendizaje de las relaciones eológicas y evolutivas.

hasta los diferentes linajes -ramas del árbol evolutivo- de las que en la actualidad conocemos escasos representantes, plantea la necesidad de elaborar metodologías que permitan establecer una interrelación entre estos, asi como con las especies actuales.

Con este proyecto se pretende implementar un material y una metodología apropiados para la enseñanza y divulgación de las mencionadas relaciones, mediante un método lúdico, atractivo a la vez que razonablemente intuitivo.

Palabras clave: Ecosistemas mediterráneos, Relaciones tróficas, Relaciones evolutivas, depredador, parasitoide, plaga, líneas evolutivas de Tetrápodos, formación, metodología.

\section{Introducción}

Los ecosistemas son hábitats autosuficientes donde los organismos vivos y el ambiente interaccionan para intercambiar energía y materia en un ciclo continuo. Las diversas e intricadas interacciones entre los organismos, y los múltiples flujos por los que se canaliza este intercambio (cadenas tróficas), hace de la divulgación científica del funcionamiento de los ecosistemas un auténtico desafío. A pesar de que estas interacciones constituyen la esencia de los ecosistemas, nunca se ha abordado la creación de un material didáctico apropiado para visualizarlas, sea tanto para fines meramente divulgativos, como para su aplicación en las Enseñanzas Media y Superior. En este último caso, son muy diversas las asignaturas en las que se aborda esta temática, entre las que citaremos como ejemplo: Entomología Aplicada, Ecología, Gestión de zona Litoral, Entomología Forestal, Entomología Agrícola, Protección Vegetal, Vertebrados, Invertebrados,..etc. incluidas en planes de estudios de Titulaciones Universitaria Superior (Licenciatura/Grado) en CC. Biológicas, CC. Ambientales, o bien en Titulaciones tanto de Escuelas Técnicas como Superior en Ingenierías Agrarias y Forestales.

En lo que respecta a la variación temporal de la Diversidad Animal, los múltiples eventos, geológicos y bioclimáticos ocurridos en el planeta, han proporcionado el motor necesario para impulsar los cambios evolutivos, marcado una dirección y un ritmo en la variación, y proporcionando, según las circunstancias ambientales, unas condiciones más propicias para la selección de una u otra línea filogenética. La variación gradual de la complejidad estructural y de la diversidad a lo largo del tiempo, y en particular en los Vertebrados, desde formas basales relativamente simples (Paleozoico Inferior), hasta los diferentes linajes -ramas del árbol evolutivo- de las que en la actualidad solo conocemos el resultado final de este largo proceso, ha planteado la necesidad de elaborar materiales didácticos específicos y metodologías asequibles para su adecuada comprensión. Dentro del contexto universitario, estos conocimientos se abordan en Titulaciones del Grado en CC Biológicas,

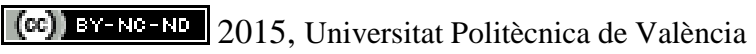


asi como en CC Ambientales, siendo tratados en asignaturas tales como: Evolución Animal, Diversidad Animal y Evolución, Diversidad Animal y Vegetal, Vertebrados y Zoología.

\section{Objetivos}

Se han pretendido alcanzar los siguientes objetivos:

\section{a/ Ecopuzzles}

El propósito principal es contribuir significativamente a la comprensión del funcionamiento de los ecosistemas, como elemento de partida para la sensibilización ambiental. Para ello, se ha procurado aportar un método sencillo, eficaz e intuitivo para el aprendizaje de las relaciones ecológicas entre especies concretas en diferentes tipos de hábitats. La visión del ecosistema como un conjunto, facilitaría el conocimiento del papel ejercido por especies animales y vegetales concretas, y de sus diferentes tipos de interacción (depredación, simbiosis, fitofagia, descomposición...) lo que a su vez debería permitir una mejor comprensión, y una mejor valoración de la importancia de estas interacciones (adecuado reciclaje de la materia orgánica, sostenibilidad de la explotación de los ecosistemas agrícolas y forestales, ..etc).

Surge como respuesta a la escasez de material didáctico y de metodologías apropiadas para las actividades divulgativas, cada vez mas demandadas por la sociedad a las universidades (Semana de la Ciencia, Noche de los Investigadores, Jornada de puertas abiertas...). Se ha buscado el componente lúdico, para asi hacerlo atractivo a todos todos los niveles, sin necesidad de conocimientos previos por parte de los usuarios. De este modo se espera fomentar y desarrollar la "memoria visual", facilitando el aprendizaje y la identificación de las especies seleccionadas (indicadores ecológicos, de importancia económica como plagas agrícolas, forestales, o de interés doméstico, industrial...etc).

\section{b/ Evopuzzles}

Al igual que en el caso anterior, este proyecto surge como respuesta a la escasez de material didáctico específico, pretendiendo por tanto cubrir, si bien en menos en parte esta laguna, aportando una metodología asequible e intuitiva, capaz de facilitar la transmisión de una información de gran actualidad. Dado que los contenidos abordados proceden en su mayor parte de investigaciones llevadas a cabo en las décadas mas recientes, y a la vista del impacto en la opinión pública en general, se ha procurado aplicar un metodología sencilla, intuitiva y asequible para cualquier nivel.

En esta obra se tratan todas las líneas evolutivas de los Vertebrados Tetrápodos (Anfibios, Reptiles, Aves y Mamíferos), proporcionando una visión espacial suficientemente completa en cuanto a variedad de formas, con el objeto de:

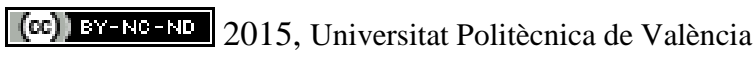

Congreso In-Red (2015): 
Ecopuzzles/Evopuzzles: una nueva metodología activa para la enseñanza-aprendizaje de las relaciones eológicas y evolutivas.

- Caracterizar la paleofauna de los diferentes periodos geológicos, visualizando las notables variaciones que han tenido lugar en la Diversidad Animal.

- Evaluar el impacto que los principales eventos bioclimáticos han tenido en los principales hitos evolutivos y en las variaciones de la Diversidad Animal. En particular, el provocado por las 4 de las 6 grandes Extinciones Masivas que han tenido lugar en la Tierra desde la aparición de los Tetrápodos hasta nuestros días (Devónico/Pérmico; Pérmico/Triásico; Triásico/Jurásico y Cretácico/Paleoceno).

- Posibilitar el conocimiento de los taxones concretos de animales prehistóricos que poblaron la Tierra, situándolos en un contexto geográfico y cronológico. Con esta integración se pretende estimular la capacidad deductiva.

- El sistema bidimensional basado en la participación activa, y planteado a modo de juego de mesa tiene como objeto estimular y ejercitar la memoria visual, facilitándoles la asimilación y adecuada organización espacio-temporal de los conocimientos.

\section{Desarrollo de la innovación}

\section{I/ Descripción genérica.}

Básicamente, se ha coemzado por seleccionar los elementos y las relaciones más destacadas que tienen lugar en los ecosistemas (Ecopuzzles), o las especies clave especialmente relevantes en la evolución animal (Evopuzzles), representándolas mediante un modelo bidimensional, basado en figuras geométricas (cuadradas-fijas y octogonalesmóviles). El adecuado ensamblaje permite acceder a la información objeto.

Se tratan aquí los ecosistemas / series evolutivas como un diagrama bidimensional representado en un tablero, y a las especies integrantes como piezas de un puzle, cuya posición y contenido permite visualizar el tipo de interacción entre las especies contiguas (Ecopuzzles), así como las relaciones evolutivas de los linajes de los vertebrados tetrápodos (Evopuzzles), para lo que se utilizan iconos que permiten situar las fichas en una determinada y única posición correcta.

Cada ficha octogonal se encuentra en contacto con 4 cuadrangulares, a la vez que con otras 4 del mismo tipo. Los octógonos, dependiendo del modelo en cuestión, corresponden fichas específicas en las que se recoge diversa información de la especie representada: biología, ecología, etología...

Las piezas cuadrangulares permiten el engarce de las anteriores, y corresponden a distintas categorías, propiedades o características sobre la ecología de las especies tratadas, o bien representan especies botánicas concretas (en función del modelo), con las que se relacionan los animales (piezas octogonales). Los distintos grupos de organismos tratados en ellas se representan en su zona central.

(c)) EY-NC-ND 2015, Universitat Politècnica de València 


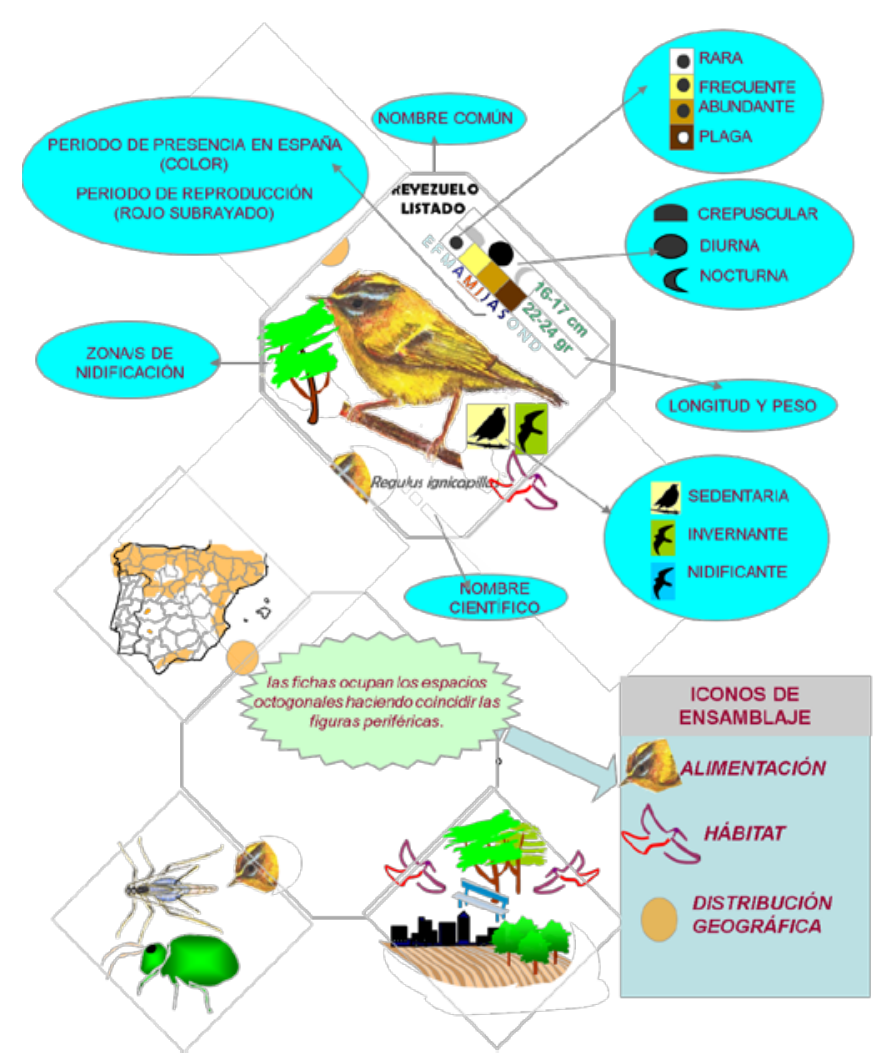

Fig. 1 Ejemplo ilustrativo de la información contenida en las fichas octogonales, y de los criterios para su adecuada colocación en el tabler (Ecopuzzle: "Aves de las ciudades”)

Se trata de encontrar las correspondencias entre las piezas, para lo que se utilizan iconos representativos de diversos aspectos (biología, ecología, alimentación, estatus, distribución...). Estos se encuentran en las zonas periféricas de las fichas, en su zona de contacto, por lo que permiten establecer una relación lógica entre octógonos-cuadrados así como entre los octógonos contiguos. Bien colocados se obtiene una visión de conjunto, y acceso a datos concretos sobre aspectos animales (geográficos, ecológicos, etológicos), así como de las interacciones particulares con otros elementos de los ecosistemas (Ecopuzzles), asicomo sobre aspectos taxonómicos, filogenéticos, paleogeográficos (Evopuzzles).

\section{II/ Descripción específica}

\section{A- Serie: Relaciones ecológicas: “Ecopuzzles”. (I) Relaciones Ecológicas en el Olivar.}

Se han seleccionado las principales especies animales del olivar, con la finalidad de dar a conocer las principales plagas y sus enemigos naturales.

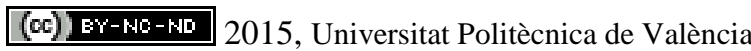


Ecopuzzles/Evopuzzles: una nueva metodología activa para la enseñanza-aprendizaje de las relaciones eológicas y evolutivas.

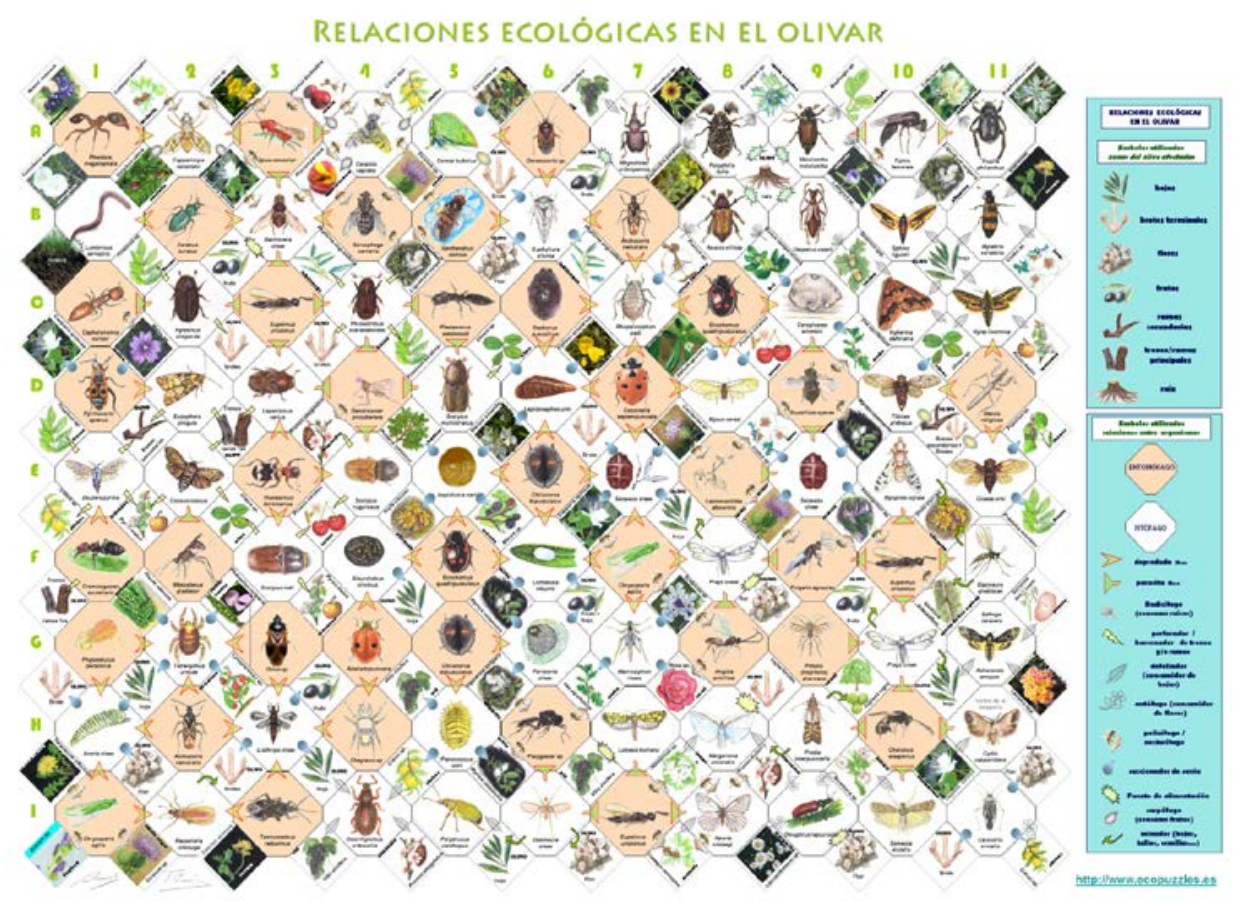

Fig. 2. Ecopuzle: "Relaciones eológicas en elolivar"

Se indica el papel que las diferentes especies herbáceas, arbustivas, arbóreas ejercen en este cultivo, ilustrando su papel beneficioso en el control natural.

\section{(II) Plagas de las Masas Forestales Españolas.}

Se tratan las especies plaga de las principales especies arbóreas de nuestras masas forestales. Se muestra el modo en que los distintos estadios de desarrollo (larva/ninfa/adulto) de estas especies interactúan con ellas. Las plagas tratadas se clasifican en función del tipo de alimentación (defoliadores, perforadores, radicífagos, chupadores de savia, antófagos, formadores de agallas...).

\section{(III) Relaciones Ecológicas en Ecosistemas Mediterráneos.}

En este se considera una selección de artrópodos característicos de ecosistemas agrícolas y forestales del bioma mediterráneo. En las especies fitófagas, se representan aspectos como tipo de fitofagia. Se describen todas y cada una de las fuentes de alimentación que les proporcionan las plantas (savia, polen, flor, hojas, fruto, tronco, raíz...). En el caso de las entomófagas, se indican sus presas potenciales y el tipo de entomofagia. 


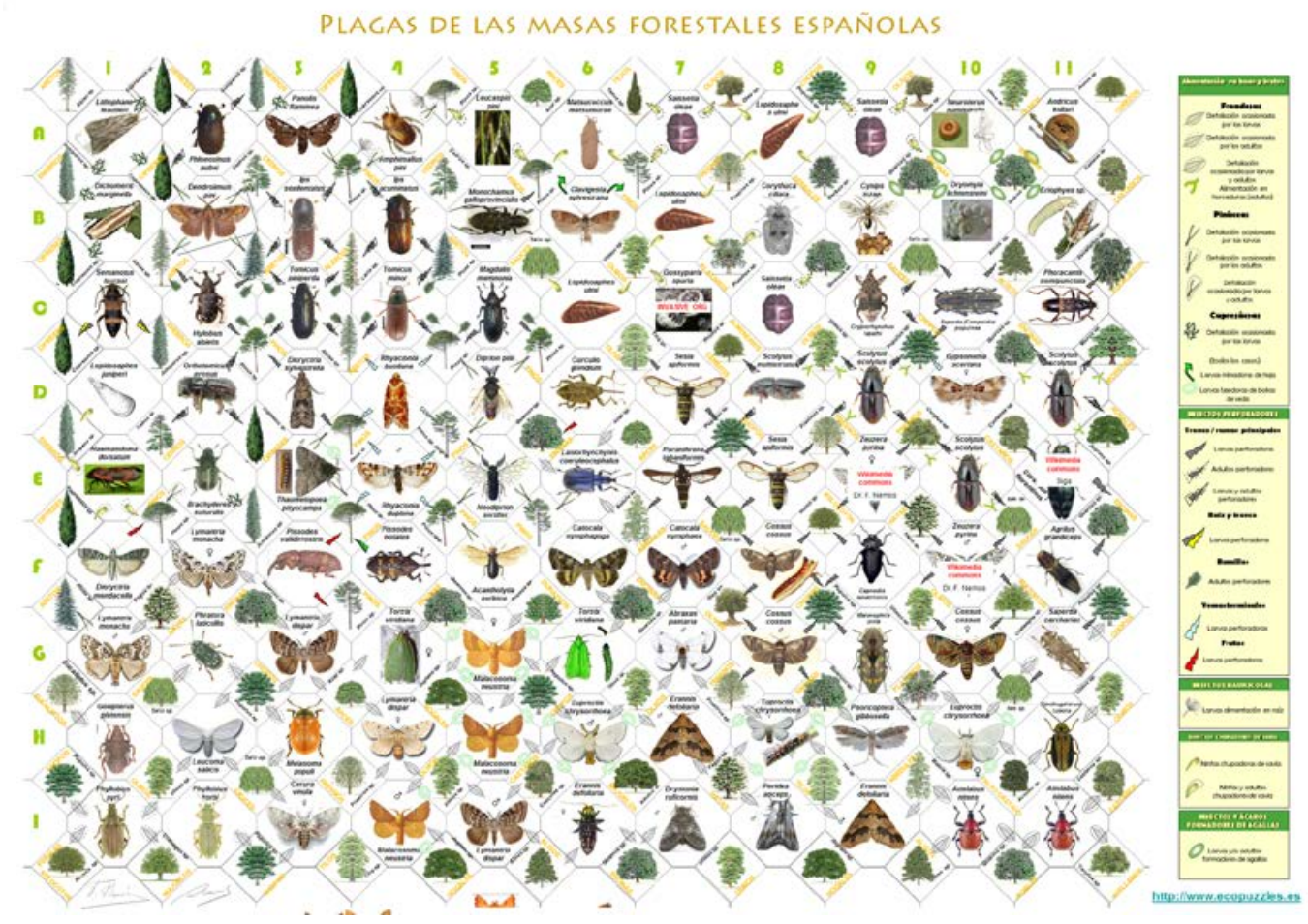

Fig. 3. Ecopuzle: "Plagas de las masas forestales españolas”

\section{(IV) Aves del Olivar.}

Las fichas indican los aspectos biológicos más relevantes de las principales especies de aves que habitan o visitan el olivar (sur de España). Se indican hábitos migratorios (sedentarias, migratorias invernales, migratorias nidificantes), abundancia relativa, actividad, época de presencia, de reproducción... Las fichas octogonales se colocan en el tablero en función del tipo de alimentación y de las zonas de nidificación.

\section{(V) Peces del Litoral Europeo.}

Se representan las principales especies de interés comercial en el litoral europeo Atlántico y Mediterráneo. El objetivo es facilitar el conocimiento de la biología y ecología de estas especies, proporcionando un material en el que se recoge información esencial para su conservación. Se indican datos biométricos (rango de peso y tamaño de los adultos), y 
Ecopuzzles/Evopuzzles: una nueva metodología activa para la enseñanza-aprendizaje de las relaciones eológicas y evolutivas.

legales (tallas mínimas; fecha de pesca autorizada).

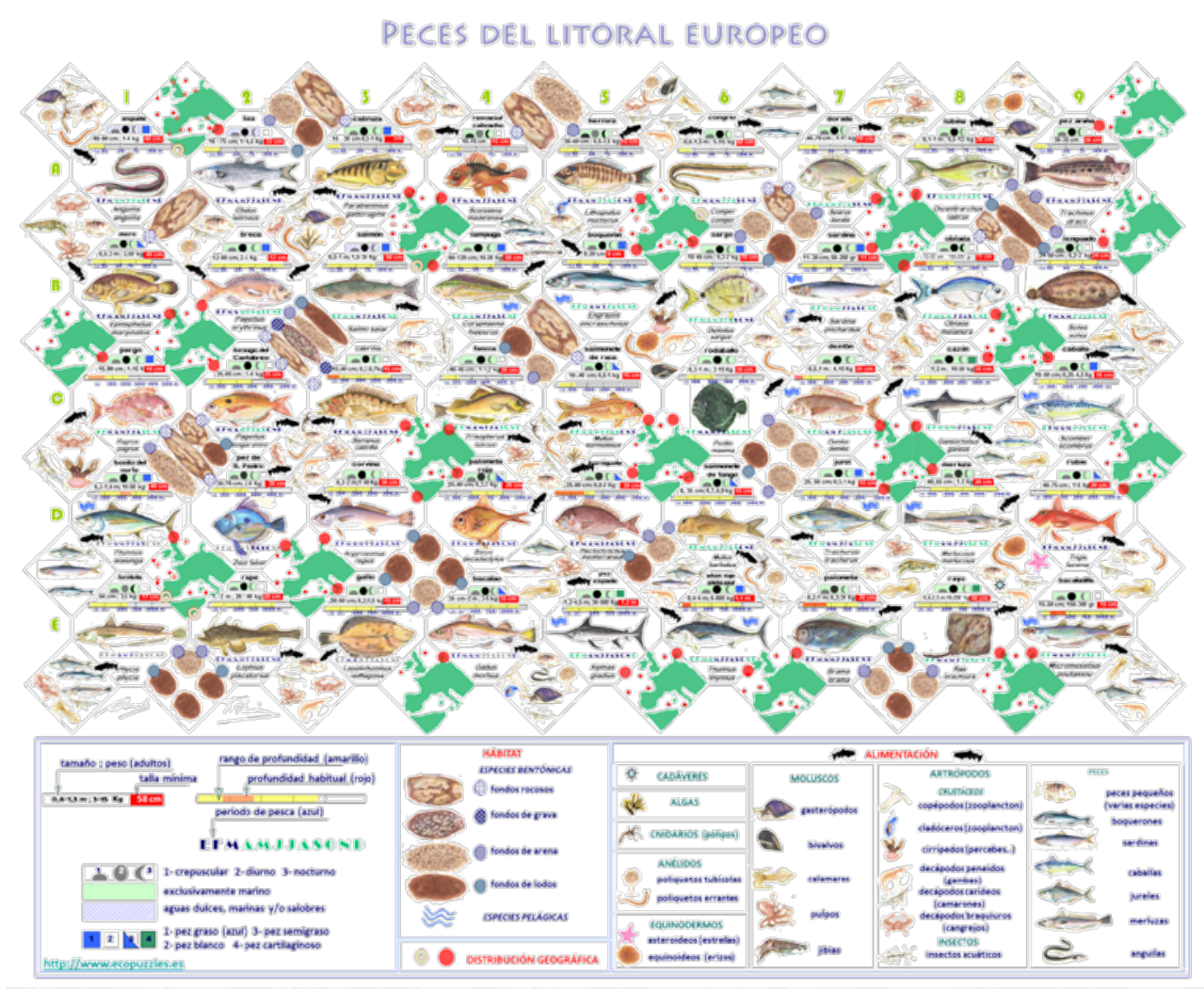

Fig. 4. Ecopuzle: "Peces del litoral europeo"

Se considera el tipo de fondo (grava, roca, arena) en el que viven, así como tipo de alimentación y distribución geográfica en el litoral europeo, tipo de actividad (crepusculares, nocturnos o diurnos), y hábitat (bentónicos, pelágicos, rango de profundidad, adaptaciones a zonas salobres y/o dulceacuícolas).

\section{(VI) Aves de las Ciudades.}

El objetivo es facilitar un material apropiado para etapas preliminares del conocimiento de las aves, considerándose aquí una selección de especies relativamente comunes en las ciudades y zonas periurbanas de la Península Ibérica.

Se presentan datos biométricos (tamaño, peso) atividad (nocturnas, crepusculares, diurnas) índice de abundancia... Para las migratorias se indica la época del año en la que permanecen en las ciudades españolas, fechas y zonas de reproducción.

(cc) EY-NC-ND 2015, Universitat Politècnica de València 


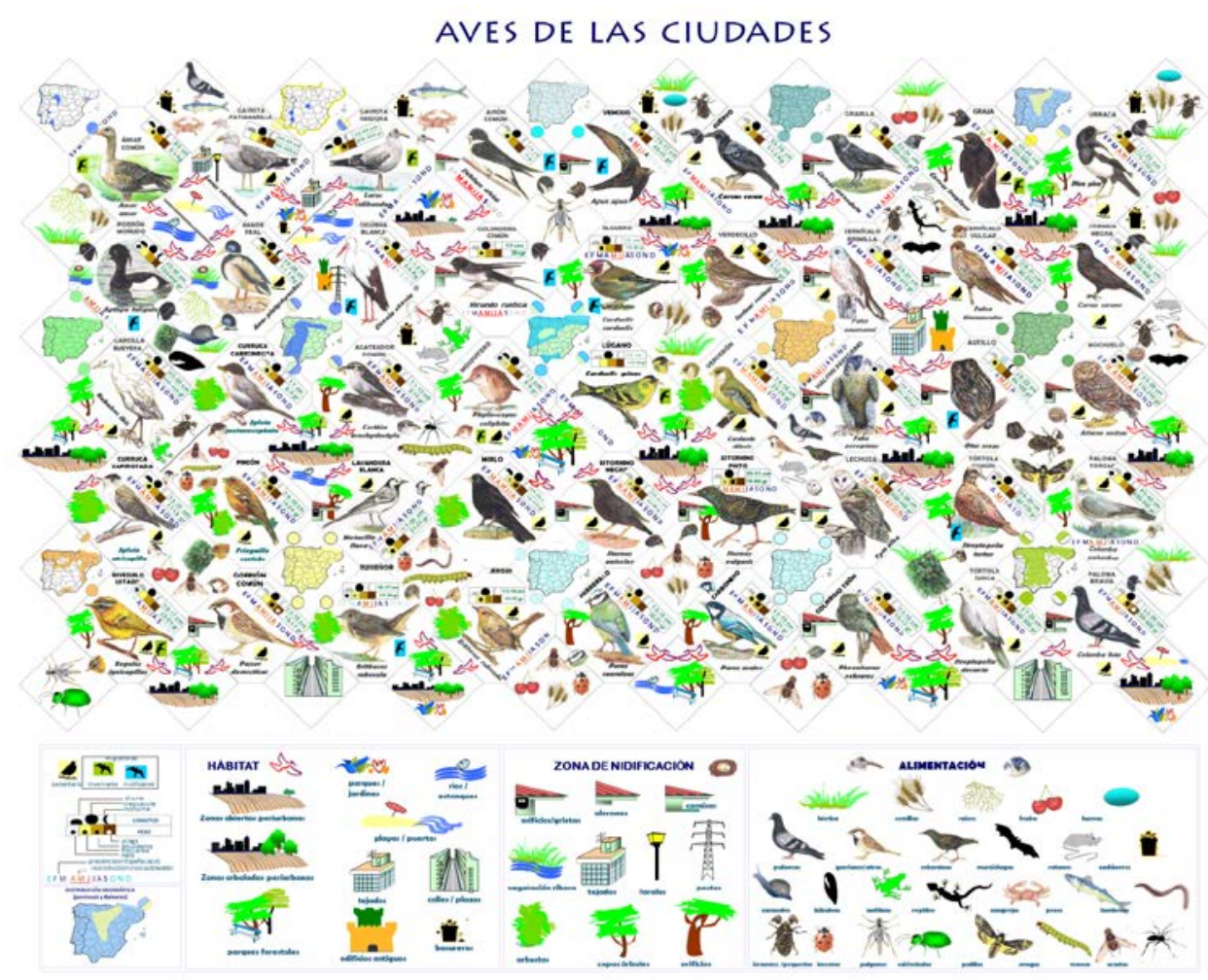

Fig. 5. Ecopuzle: "Aves de las ciudades”

Las fichas se sitúan atendiendo a factores como: hábitat o alimentación, indicándose mediante iconos las fuentes alimenticias más usuales, y su distribución geográfica.

\section{B. Serie: Relaciones evolutivas: “Evopuzzles” http://www.evopuzzles.es}

(I) Paleozoico: Devónico/Pérmico (416/251 M.a.).

Los Tetrápodos aparecen hacia la segunda mitad del Paleozoico, procedentes de linajes de peces óseos de hábitos anfibios (Sarcopterigios). Su diversificación fue posible gracias a la desaparición de los grandes depredadores acuáticos, los Placodermos, causada por la segunda gran Extinción Masiva (finales del Devónico, 350 m.a.). La conquista del medio terrestre, y la la huida de los de los hábitats acuáticos que se han tornado inhóspitos, representa el mayor salto evolutivo que ha tenido lugar en el planeta. Se representan las distintas líneas evolutivas (Sarcopterigios y Anfibios).

Igualmente refleja el segundo evento más importante en la evolución de los Tetrápodos, la aparición del amnios (Carbonífero), y con ello la independencia de los hábitats acuáticos, con la llegada de los primeros Reptiles. Estos serán por tanto los dominantes hasta la siguiente extinción, que tendría lugar a finales del período Pérmico.

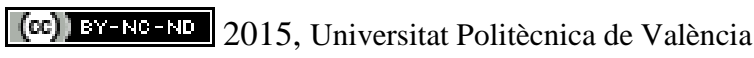

Congreso In-Red (2015): 
Ecopuzzles/Evopuzzles: una nueva metodología activa para la enseñanza-aprendizaje de las relaciones eológicas y evolutivas.

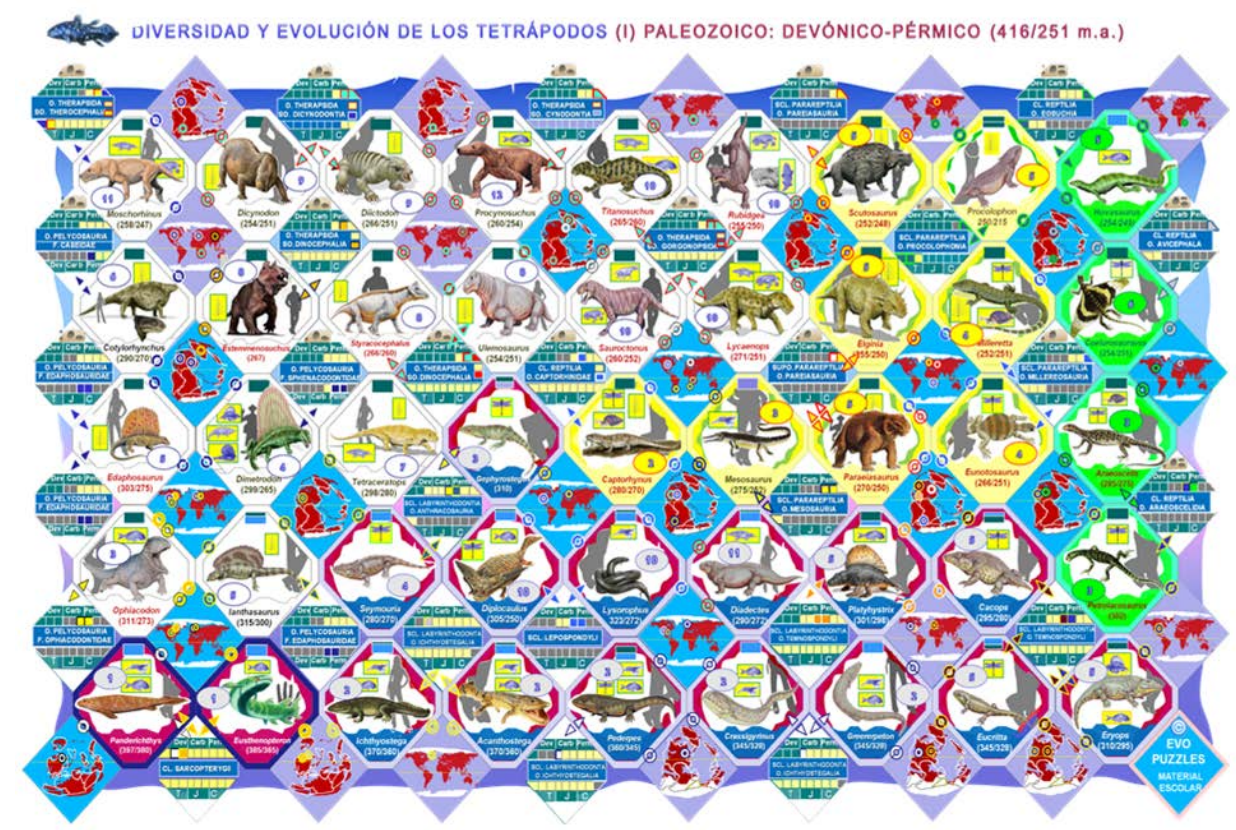

Fig. 6. Evopuzle: “Diversidad y evolución de los Tetrápodos (I): Paleozoico”

(II) Mesozoico: Triásico (251/199 M.a.).

A fines del Pérmico tiene lugar la Tercera Gran Extinción masiva (250 M.a.) desencadenando un drástico cambio en la Diversidad Animal. Los reptiles de la línea Sinápsidos, antes dominantes, sufren especialmente los efectos. Ello proporciona no obstante la posibilidad evolutiva para la hasta ahora minoritaria línea de reptiles Diápsidos. De estos surgirán diferentes líneas que conducirán a los Dinosaurios, Plesiosaurios, Pterosaurios, Ictiosáurios, Cocodrilos, así como a los ancestros de las Aves, mejor adaptados, anatómica y fisiológicamente, para afrontar las nuevas condiciones.

\section{(III) Mesozoico: Jurásico (199/145 M.a.).}

Aparecen los Anfibios del tipo actual. Se diversifican los Dinosaurios (en el Triásico eran de reducidas dimensiones), evolucionando rápidamente, y siendo dominantes en los ecosistemas terrestres. Se diferencian las dos líneas: Saurisquios y Ornitisquios (herbívoros como Stegosaurus), y entre los primeros se diferencian los Saurópodos (herbívoros como Diplodocus, Brachiosaurus) y los Terópodos carnívoros como Allosaurus.

Aparecen las Aves, aunque son los Pterosaurios quienes dominaban el medio aéreo, y Plesiosaurios e Ictiosaurios, los hábitats marinos.

Especialmente significativa fue la aparición de los primeros Mamíferos, de muy reducidas dimensiones, y limitados a hábitats subterráneos y hábitos nocturnos.

(c)) EY-NC-ND 2015, Universitat Politècnica de València Congreso IN-RED (2015): 


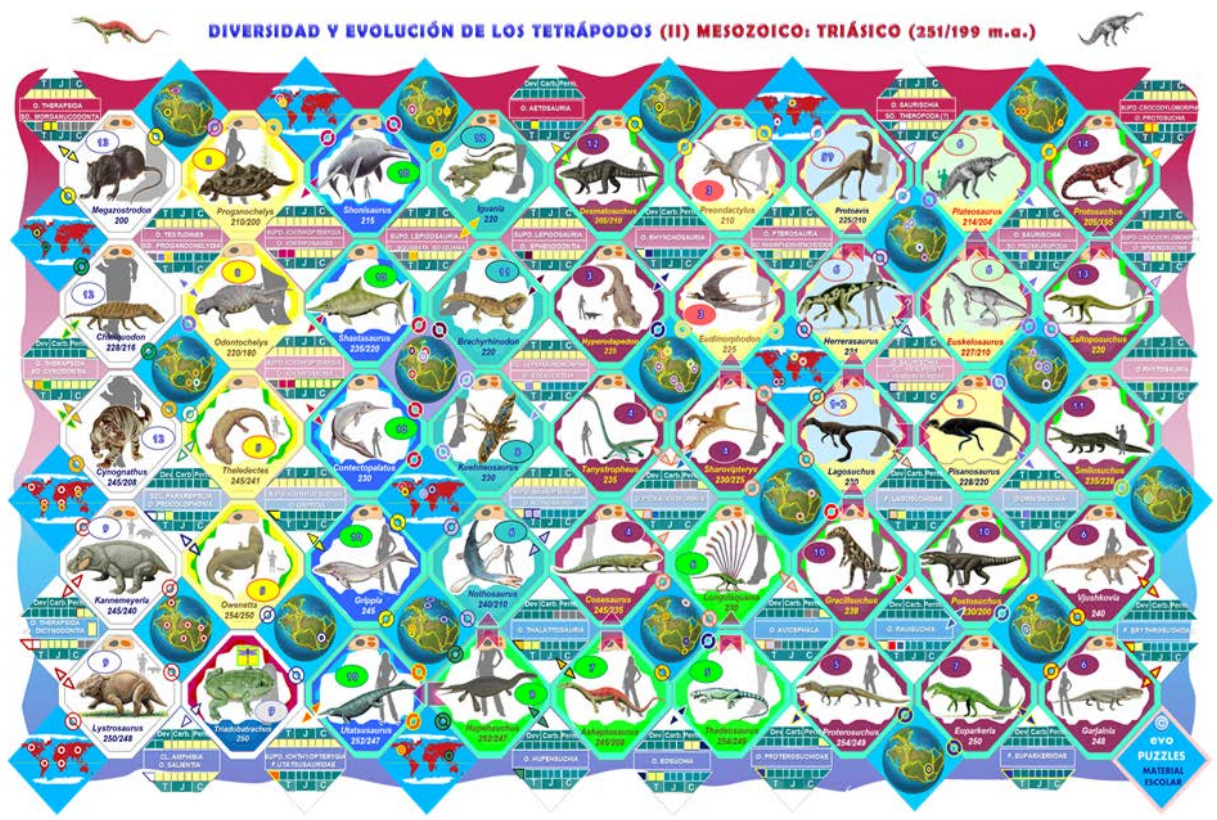

Fig. 7. Evopuzle: "Diversidad y evolución de los Tetrápodos (II): Triásico”

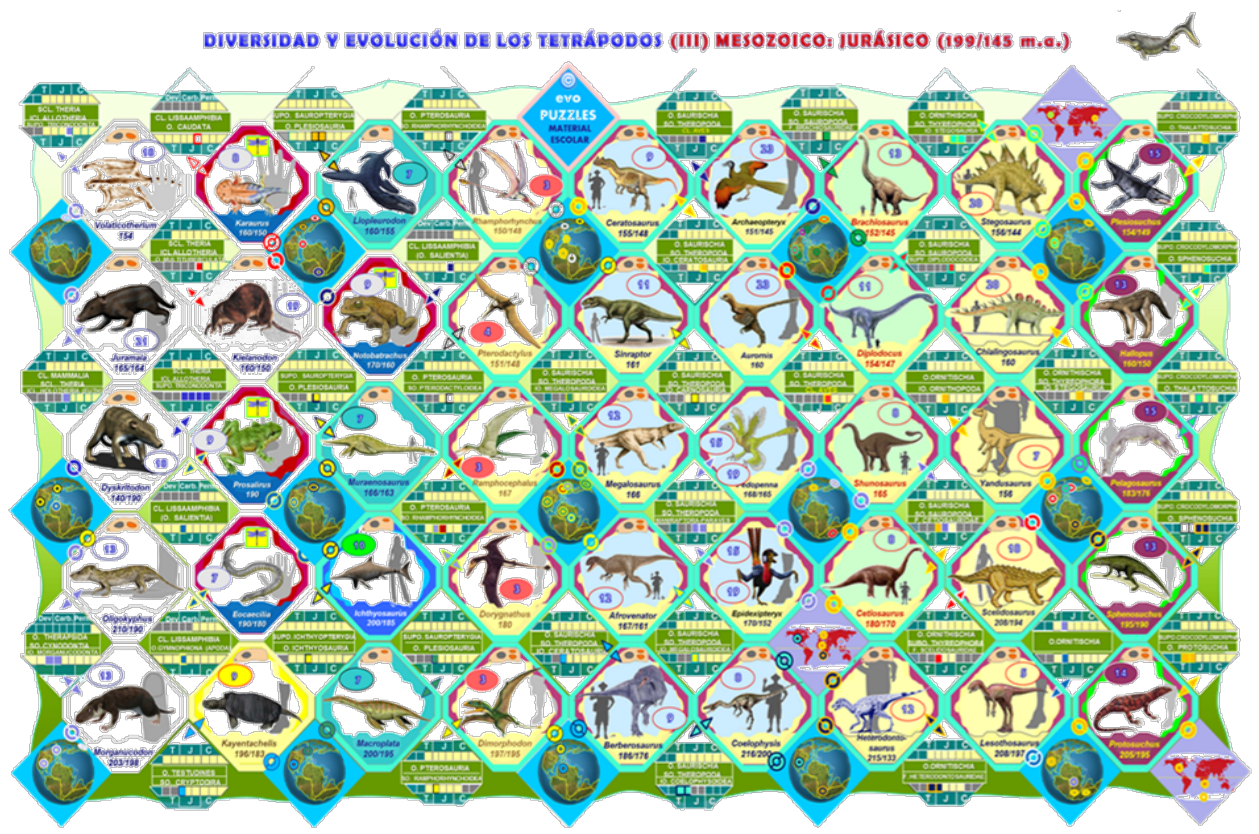

Fig. 8. Evopuzle: "Diversidad y evolución de los Tetrápodos (III): Jurásico"

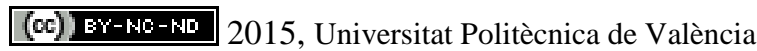

Congreso In-Red (2015): 
Ecopuzzles/Evopuzzles: una nueva metodología activa para la enseñanza-aprendizaje de las relaciones eológicas y evolutivas.

(IV) Mesozoico: Cretácico (145/65 M.a.).

Se ilustran aqui los cambios mas significativos que tuvieron lugar en este periodo:

En el mar desaparecen los Ictiosaurios, sustituidos por grandes Condríctios (tiburones), peces óseos (Teleósteos), y grandes tortugas (Archelon), susbsistiendo aún los Plesiosaurios (Elasmosaurus). Irrumpen los Lepidosaurios marinos como Mosasaurus, el mayor predador marino a finales del Cretácico, emparentado con lagartos y serpientes, las cuales ya comienzan a despuntar desde mediados de este período (Pachyrhachis).

En tierra continúan diversificándose los Ornitisquios bípedos, muy abundantes (Iguanodon y Parasaurolophus) dotados de mandíbulas y genas que les permiten una más eficaz masticación. Entre los Saurópodos aparecen las especies de gran tamaño (hasta $40 \mathrm{~m}$ como Argentinosaurus, Turiasaurus..) y entre los Terópodos carnívoros de toda gama de tamaños, desde el Velociraptor hasta los grandes Tyrannosaurus rex y Tarbosaurus.

En el aire continúan los Pterosaurios (Pteranodon). Las aves son escasas y minoritarias (Ichthyornis), aunque comienzan a adaptarse al hábitat acuático (Hesperornis).

Los Mamíferos son un componente minoritario de la fauna, relegados a hábitats subterráneos y arborícolas (Purgatorius). No obstante, el impacto del asteroide (5 gran Extinción Masiva), marcó el declive de los Dinosaurios, y la inversión ecológica posterior, por cuanto a comienzos de la Era Terciaria serán inicialmente las Aves, y posteriormente los Mamíferos, los grupos que se disputarían el papel dominante.

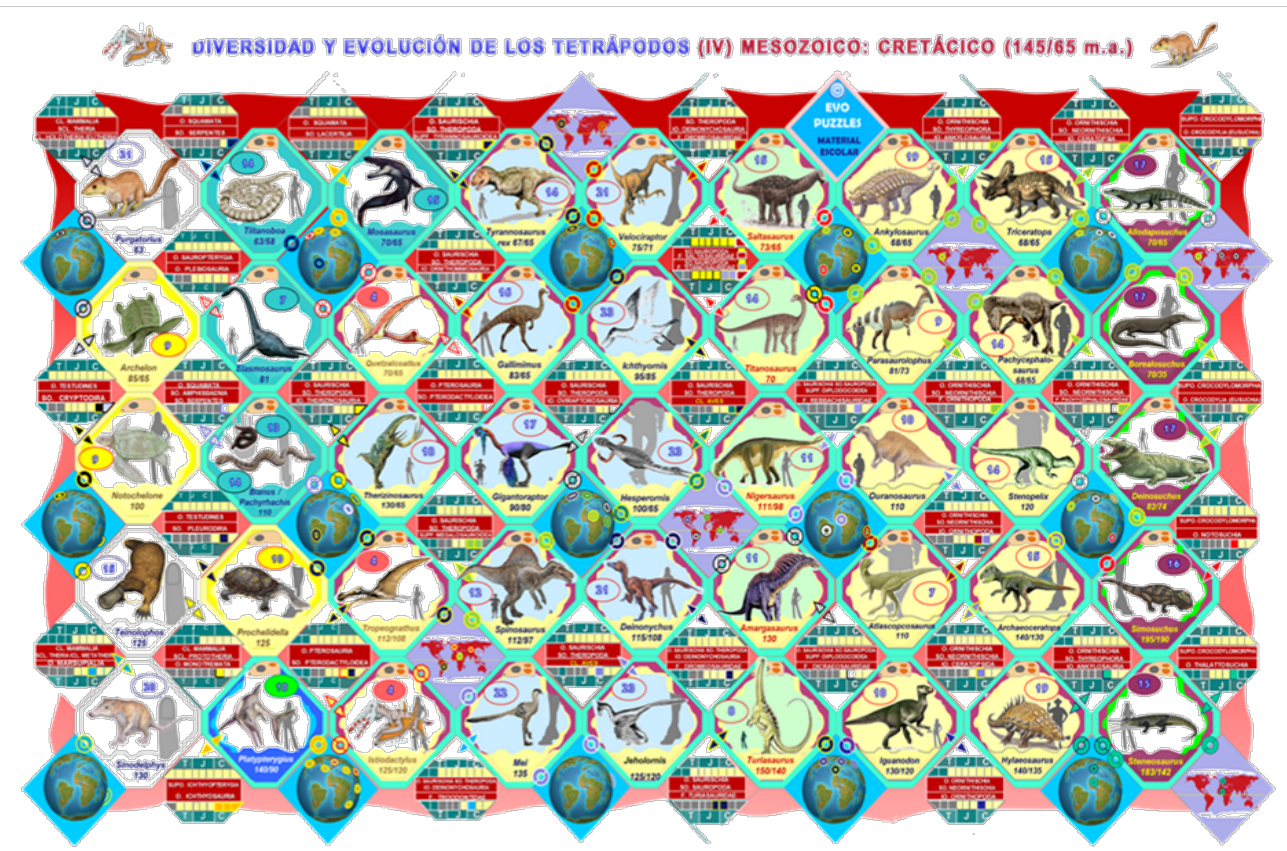

Fig. 9. Evopuzle: “Diversidad y evolución de los Tetrápodos (III): Cretácico” 


\section{Resultados}

La actividades prácticas descritas han sido llevadas a cabo en diversas ocasiones, incuidas en diversas jornadas de divulgación organizadas por parte de los Vicerrectorados de Planificación, Calidad, Responsabilidad Social y Comunicación, y de Investigación, Desarrollo Tecnológico e Innovación, previamente adaptado a un nivel básico. Igualmente, se ha aplicado recientemente como material de apoyo en enseñanza Superior de la Universidad de Jaén, en sus niveles medio y avanzado.

\section{A. Nivel básico:}

"Semana de la Ciencia" (2010, 2011, 2012, 2013, 2014).

"Jornadas: Ciencia e Innovación en Ruta, 2013" (Colegios de la provincia de Jaén).

"Renaciencia" (Úbeda Jaén 2014).

“Noche de los Investigadores” (Jaén, 2013).

Algunos enlaces que hacen referencia a la puesta en práctica de estas actividades:

http://www.andaluciainformacion.es/portada/?a $=150049 \& \mathrm{i}=35 \& \mathrm{f}=0$

http://www.ideal.es/jaen/v/20101109/jaen/enganchar-jovenes-ciencia-20101109.html

http://diariodigital.ujaen.es/node/26892

http://www.diariojaen.es/index.php/menujaen/25-notlocales/28352-comienza-la-x-semana-de-

la-ciencia

http://diariodigital.ujaen.es/node/33422

www.cope.es/detalle/universidad-.html

http://diariodigital.ujaen.es/node/37283

http://diariodigital.ujaen.es/node/26919

http://diariodigital.ujaen.es/node/40981

http://noticias.lainformacion.com/ciencia-y-tecnologia/biologia/la-uja-participa-en-ubeda-enlas-jornadas-ciencia-para-todos-con-la-actividad-divulgativaecopuzles_Mtswsy9ybaxOR6XjiACQP/

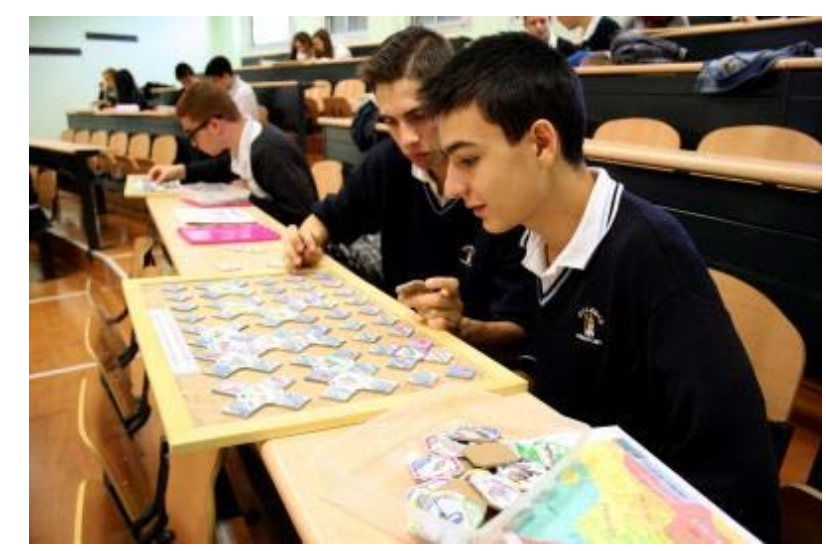

Fig.10. Desarrollo de la actividad (alumnos del Colegio Cristo Rey, Jaén).

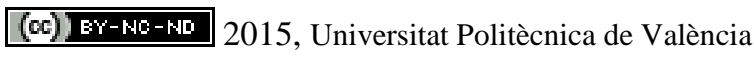

Congreso In-Red (2015): 
BENTON, M. J. (2006). “The origin of the Dinosaurs” Colectivo Arqueológico-Paleontológico Salense, Ed. en Actas de las III Jornadas sobre Dinosaurios y su Entorno. 11-19. Salas de los Infantes, Burgos, España, 12 pp.

DZIK, J. (2003). “A beaked herbivorous archosaur with dinosaur affinities from the early Late Triassic of Poland” en Journal of Vertebrate Paleontology, 23(3): 556-574.

FERIGOLO, J. y LANGER, M.C. (2006). "A Late Triassic dinosauriform from south Brazil and the origin of the ornithischian predentary bone" en Historical Biology 19 (1): 1-11.

GUILLON, J-M., GUÉRY, L.; HULIN, V. y GIRONDOT, M. (2012). “A large phylogeny of turtles (Testudines) using molecular data” en Contributions to Zoology, 81 (3) 147-158 (2012)

IRMIS, R. B., PARKER, W. G., NESBITT, S. J. y LIU, J. (2007). "Early ornithischian dinosaurs: the Triassic record" en Historical Biology, 2007; 19(1): 3-22

IRMIS, R. B., PARKER, W. G., SMITH, N. D., TURNER, A. H. y ROWE, T. (2009). "Hindlimb osteology and distribution of basal dinosauromorphs from the Late Triassic of North America" en Journal of Vertebrate Paleontology 29 (2): 498-516.

LANGER, M.C., ABDALA, F., RICHTER, M., y BENTON, M. (1999). "A sauropodomorph dinosaur from the Upper Triassic (Carnian) of southern Brazil" en Comptes Rendus de l'Académie des Sciences, 329: 511-;517.

MARTÍNEZ, R. N. y ALCOBER, O. A. (2009). “A Basal Sauropodomorph (Dinosauria: Saurischia) from the Ischigualasto Formation (Triassic, Carnian) and the Early Evolution of Sauropodomorpha” en Plos One, vol. 4, issue 2 E4397.

NESBITT, S. J.; SMITH, N. D.; IRMIS, R. B., TURNER, A. H., DOWNS A. y NOREL M. A. (2009). “A complete Skeleton of Late Triassic Saurischian and Early Evolution of Dinosaurs" en Science, vol 326. 11.12.2009

NOVAS F.E. (1994). “Origen de los Dinosaurios” en Investigación y Ciencia, $N^{\circ}$ 217: 52-59.

RAUHUT, O.M.W. y HUNGERBÜHLER. (2000). "A review of European Triassic theropods" en Gaia, 15: 75-88.

1997. 25:435-89.

STERLI, J. (2010). "Phylogenetic relationships among extinct and extant turtles: the position of Pleurodira and the effects of the fossils on rooting crown-group turtles” en Contributions to Zoology, 79 (3) 93-106 (2010). 